\title{
A problem of Galambos on Engel expansions
}

\author{
by
}

\section{JuN Wu (Wuhan)}

1. Introduction. Given $x$ in $(0,1]$, let $x=\left[d_{1}(x), d_{2}(x), \ldots\right]$ denote the Engel expansion of $x$, that is,

$$
x=\frac{1}{d_{1}(x)}+\frac{1}{d_{1}(x) d_{2}(x)}+\ldots+\frac{1}{d_{1}(x) d_{2}(x) \ldots d_{n}(x)}+\ldots,
$$

where $\left\{d_{j}(x), j \geq 1\right\}$ is a sequence of positive integers satisfying $d_{1}(x) \geq 2$ and $d_{j+1}(x) \geq d_{j}(x)$ for $j \geq 1$. (See [3].) In [3], János Galambos proved that for almost all $x \in(0,1]$,

$$
\lim _{n \rightarrow \infty} d_{n}^{1 / n}(x)=e .
$$

He conjectured ([3], P132) that the Hausdorff dimension of the set where (2) fails is one. In this paper, we prove this conjecture:

Theorem. $\operatorname{dim}_{H}\{x \in(0,1]:(2)$ fails $\}=1$.

We use $L^{1}$ to denote the one-dimensional Lebesgue measure on $(0,1]$ and $\operatorname{dim}_{\mathrm{H}}$ to denote the Hausdorff dimension.

2. Proof of Theorem. The aim of this section is to prove the main result of this paper.

By Egoroff's Theorem, there exists a Borel set $A \subset(0,1]$ with $L^{1}(A)$ $\geq 1 / 2$ such that $\left\{d_{n}^{1 / n}(x), n \geq 1\right\}$ converges to $e$ uniformly on $A$. In particular, there exists a positive number $N$ such that

$$
2 \leq d_{n}^{1 / n}(x) \leq 3 \quad \text { for all } n \geq N \text { and } x \in A .
$$

Choose a positive integer $M$ satisfying $M \geq N$. For any $x=\left[d_{1}, d_{2}, \ldots\right]=$ $\left[d_{1}, d_{2}, \ldots, d_{M}, d_{M+1}, d_{M+2}, \ldots, d_{k M+1}, d_{k M+2}, \ldots, d_{(k+1) M}, \ldots\right]$, we construct a new point $\bar{x} \in(0,1]$ as follows:

$$
\bar{x}=\left[\bar{d}_{1}, \bar{d}_{2}, \ldots\right]
$$

2000 Mathematics Subject Classification: Primary 11K55; Secondary 28A80.

Project supported by National Natural Science Foundation of China. 
where $\bar{d}_{k(M+1)+l}=d_{k M+l}$ for all $k \geq 0$ and $0 \leq l \leq M$. That is,

(4) $\bar{x}=\left[d_{1}, d_{2}, \ldots, d_{M}, d_{M}, d_{M+1}, d_{M+2}, \ldots\right.$,

$$
\left.d_{k M+1}, d_{k M+2}, \ldots, d_{(k+1) M}, d_{(k+1) M}, \ldots\right] .
$$

Lemma 1. $\{\bar{x}: x \in A\} \subset\{x \in(0,1]:(2)$ fails $\}$.

Proof. Note that for any $k \geq 1, d_{k(M+1)}(\bar{x})=d_{k M}(x)$. We have

$$
\lim _{k \rightarrow \infty} d_{k(M+1)}^{1 /(k(M+1))}(\bar{x})=\lim _{k \rightarrow \infty}\left(d_{k M}^{1 /(k M)}(x)\right)^{k M /(k(M+1))}=e^{M /(M+1)},
$$

and this proves the assertion.

For any $x=\left[d_{1}(x), d_{2}(x), \ldots\right] \in(0,1], y=\left[d_{1}(y), d_{2}(y), \ldots\right] \in(0,1]$, define

$$
\varrho(x, y)=\inf \left\{j: d_{j}(x) \neq d_{j}(y)\right\} \quad(\inf \emptyset=\infty) .
$$

For any $x, y \in(0,1], x \neq y$, suppose $\varrho(x, y)=k$. Without loss of generality, assume $d_{k}(x)<d_{k}(y)$. Then $x>y$ and $x \in(B, C], y \in(D, E]$ with

$$
\begin{aligned}
B= & \frac{1}{d_{1}(x)}+\frac{1}{d_{1}(x) d_{2}(x)}+\ldots+\frac{1}{d_{1}(x) d_{2}(x) \ldots d_{k-1}(x) d_{k}(x)} \\
& +\frac{1}{d_{1}(x) d_{2}(x) \ldots d_{k}(x) d_{k+1}(x)}, \\
C= & \frac{1}{d_{1}(x)}+\frac{1}{d_{1}(x) d_{2}(x)}+\ldots+\frac{1}{d_{1}(x) d_{2}(x) \ldots d_{k-1}(x)\left(d_{k}(x)-1\right)} \\
D= & \frac{1}{d_{1}(y)}+\frac{1}{d_{1}(y) d_{2}(y)}+\ldots+\frac{1}{d_{1}(y) d_{2}(y) \ldots d_{k-1}(y) d_{k}(y)} \\
E= & \frac{1}{d_{1}(y)}+\frac{1}{d_{1}(y) d_{2}(y)}+\ldots+\frac{1}{d_{1}(y) d_{2}(y) \ldots d_{k-1}(y) d_{k}(y)} \\
& +\frac{1}{d_{1}(y) d_{2}(y) \ldots d_{k}(y)\left(d_{k+1}(y)-1\right)},
\end{aligned}
$$

hence

(6) $\frac{1}{d_{1}(x) d_{2}(x) \ldots d_{k}(x) d_{k+1}(x)} \leq|x-y| \leq \frac{1}{d_{1}(x) d_{2}(x) \ldots d_{k-1}(x)}$,

where $d_{0}(x) \equiv 1$.

Let

$$
\varepsilon=\frac{6 \log 3}{M \log 2}, \quad c=\frac{1}{3^{4 M(M+1)}} .
$$

Lemma 2. For any $x, y \in A$,

$$
|\bar{x}-\bar{y}| \geq c|x-y|^{1+2 \varepsilon} .
$$

Pr o of. Without loss of generality, assume $x>y$. Suppose $\varrho(x, y)=k$. 
(a) If $k \leq 2 M$, then by (3), (4) and (6), we have

$|\bar{x}-\bar{y}| \geq \frac{1}{d_{1}(\bar{x}) d_{2}(\bar{x}) \ldots d_{k}(\bar{x}) d_{k+1}(\bar{x}) d_{k+2}(\bar{x})} \geq\left(\frac{1}{3^{2 M}}\right)^{2 M+2} \geq c|x-y|^{1+2 \varepsilon}$.

(b) If $p M<k \leq(p+1) M$ for some $p \geq 2$, then by (4) and (6), we have

$$
|\bar{x}-\bar{y}| \geq \prod_{j=0}^{p-1}\left[\left(\prod_{l=1}^{M} \frac{1}{d_{j M+l}(x)}\right) \frac{1}{d_{j M+M}(x)}\right] \prod_{j=p M+1}^{k+1} \frac{1}{d_{j}(x)} .
$$

For $1 \leq j \leq p-1$, by (3), we have

$$
d_{j M+M}(x) \leq 3^{j M+M} \leq 3^{2 j M} \leq\left(\prod_{l=1}^{M} 2^{j M+l}\right)^{\varepsilon} \leq\left(\prod_{l=1}^{M} d_{j M+l}(x)\right)^{\varepsilon},
$$

thus

(10) $\left(\prod_{l=1}^{M} \frac{1}{d_{j M+l}(x)}\right) \frac{1}{d_{j M+M}(x)} \geq\left(\prod_{l=1}^{M} \frac{1}{d_{j M+l}(x)}\right)^{1+\varepsilon}, \quad 1 \leq j \leq p-1$.

For $j=0$,

$$
\left(\prod_{l=1}^{M} \frac{1}{d_{l}(x)}\right) \frac{1}{d_{M}(x)} \geq \frac{1}{3^{M(M+1)}} .
$$

On the other hand,

$$
\begin{aligned}
d_{k}(x) d_{k+1}(x) & \leq 3^{2 k+1} \leq 3^{3 k} \leq\left(2^{M} 2^{M+1} \ldots 2^{k-1}\right)^{\varepsilon} \\
& \leq\left(d_{M}(x) \ldots d_{k-1}(x)\right)^{\varepsilon}
\end{aligned}
$$

hence

$$
\frac{1}{d_{k}(x) d_{k+1}(x)} \geq\left(\frac{1}{d_{1}(x) d_{2}(x) \ldots d_{k-1}(x)}\right)^{\varepsilon} .
$$

Combining (10), (11) and (13), we have

$$
\begin{aligned}
& \geq \frac{|\bar{x}-\bar{y}|}{3^{M(M+1)}}\left[\prod_{j=1}^{p-1}\left(\prod_{l=1}^{M} \frac{1}{d_{j M+l}(x)}\right)^{1+\varepsilon}\right]\left(\prod_{j=p M+1}^{k-1} \frac{1}{d_{j}(x)}\right) \frac{1}{d_{k}(x)} \cdot \frac{1}{d_{k+1}(x)} \\
& \quad \geq \frac{1}{3^{M(M+1)}}\left(\prod_{i=1}^{k-1} \frac{1}{d_{i}(x)}\right)^{1+2 \varepsilon} \geq c|x-y|^{1+2 \varepsilon} .
\end{aligned}
$$

Proof of Theorem. Consider a map $f: A \rightarrow(0,1]$ defined by $f(x)=\bar{x}$. Note that $f: A \rightarrow f(A)$ is bijective. Lemma 2 implies that the inverse map of $f$ is $1 /(1+2 \varepsilon)$-Hölder. By Lemma 1 and [2], Proposition 2.3, we have 


$$
\begin{aligned}
1 & =\operatorname{dim}_{\mathrm{H}} A=\operatorname{dim}_{\mathrm{H}}\left[f^{-1}(f(A))\right] \leq(1+2 \varepsilon) \operatorname{dim}_{\mathrm{H}} f(A) \\
& \leq(1+2 \varepsilon) \operatorname{dim}_{\mathrm{H}}\{x \in(0,1]:(2) \text { fails }\} .
\end{aligned}
$$

Hence

$$
\operatorname{dim}_{H}\{x \in(0,1]:(2) \text { fails }\} \geq \frac{1}{1+\frac{12}{M} \cdot \frac{\log 3}{\log 2}} .
$$

Since $M>N$ is arbitrary, we have

$$
\operatorname{dim}_{\mathrm{H}}\{x \in(0,1]:(2) \text { fails }\}=1,
$$

and this completes the proof of Theorem.

\section{References}

[1] P. Erdős, A. Rényi and P. Szüsz, On Engel's and Sylvester's series, Ann. Univ. Sci. Budapest. Eötvös Sect. Math. 1 (1958), 7-32.

[2] K. J. Falconer, Fractal Geometry: Mathematical Foundations and Applications, Wiley, 1990.

[3] J. Galambos, Representations of Real Numbers by Infinite Series, Lecture Notes in Math. 502, Springer, 1976.

[4] - , The Hausdorff dimension of sets related to g-expansions, Acta Arith. 20 (1972), 385-392.

[5] - The ergodic properties of the denominators in the Oppenheim expansion of real numbers into infinite series of rationals, Quart. J. Math. Oxford Ser. (2) 21 (1970), $177-191$.

Department of Mathematics and Center of Non-linear Science

Wuhan University

430072, Wuhan

People's Republic of China

E-mail: wujunyu@public.wh.hb.cn 